La brochure «Der HIV-Test»* n'émane ni de l'Office fédéral de la santé publique (OFSP), ni de l'Aide suisse contre le sida. Son contenu ne correspond pas

à la stratégie suisse en matière de prévention du VIH.

\title{
Attention! Veuillez lire la notice d'emballage
}

Pietro Vernazza

Médecin-chef en infectiologie / hygiène hospitalière, Hôpital cantonal de St-Gall; Président de la Commission fédérale pour la santé sexuelle (CFSS), ex-Commission fédérale pour les problèmes liés au sida (CFPS)
Correspondance: Secrétariat CFSS Luciano Ruggia OFSP Case postale CH-3003 Berne Tél. 0313240667 Fax 0313240942
La brochure publiée par l'association SIDA Information Suisse contient des propos qui ne sont pas prouvés scientifiquement, voire qui vont à l'encontre des bases scientifiques établies. Elle fait également fi de la politique suisse en matière de prévention du VIH, définie dans le Programme national VIH et autres infections sexuellement transmissibles (PNVI) 20112017. Cette brochure est sponsorisée par «medica», un laboratoire où il est possible de passer un test VIH à tout moment et sans rendez-vous. SIDA Information Suisse est souvent confondue avec l'Aide suisse contre le sida. Des dons lui permettent de diffuser encore et toujours des messages publicitaires de grande ampleur.

\section{Chacun est responsable de sa protection}

La Suisse a décidé de s'engager de manière délibérée dans la stratégie d'apprentissage, suivant ainsi celle du New Public Health. Chaque individu est responsable de se protéger du VIH lors de rapports sexuels, qu'ils soient occasionnels ou non. Le respect systématique des règles du safer sex offre une protection fiable:

1. Pas de pénétration sans préservatif

2. Pas de sperme ni de sang dans la bouche

3. En cas de démangeaison, de brûlure ou d'écoulement, consulter un médecin

\section{Un traitement ne doit avoir lieu que sur indication}

Les personnes séropositives ne doivent recevoir des médicaments que si une thérapie est indiquée. Aujourd'hui, on ne connait pas les effets secondaires à long terme du traitement antirétroviral (TAR). La déclaration générale de SIDA Information Suisse, selon laquelle une thérapie doit être commencée le plus tôt possible, n'est donc pas opportune. De plus, la bonne adhérence thérapeutique revêt une importance cruciale. Selon les recommandations de la Commission d'experts clinique et thérapie VIH/sida (CCT) [1], le début de traitement est indiqué pour tous les patients asymptomatiques présentant un nombre de cellules CD4 de 350/ $\mathrm{ll}$ et moins, et dont l'adhérence au traitement a été vérifiée.

\section{Une thérapie ne suffit pas, à elle seule, à faire disparaître l'épidémie du VIH}

Pour éliminer l'épidémie du VIH, SIDA Information Suisse indique que toutes les personnes âgées
* Cet article se réfère à une brochure envoyée avec la présente édition du BMS et constituant une annexe payée, indépendante de la partie rédactionnelle.

La version française de cette brochure a été jointe au BMS n 10 du 7 mars 2012.

entre 15 et 65 ans doivent se soumettre volontairement à un test du VIH et que les individus séropositifs doivent suivre un traitement sans tarder. Malheureusement, cette recette - (trop) simple n'est pas appropriée compte tenu des diverses réalités:

- Ce scénario, qui se base sur une conception désuète de la santé publique (détecter et éliminer la source d'infection), est stigmatisant et empiète sur la sphère intime des individus. Même si la détection précoce d'une éventuelle infection peut être profitable, plusieurs raisons individuelles s'y opposent. En Suisse, personne n'est forcé de passer un test du VIH. La contrainte entraîne une résistance et empêche une prévention efficace.

- La thérapie doit être voulue. Pour qu'un TAR soit efficace, l'adhérence thérapeutique doit être bonne, sinon une résistance au traitement risque de se développer.

- Les infections sexuellement transmissibles (IST) favorisent la transmission du VIH. Toutefois, même si tous les patients séropositifs étaient traités avec un TAR, le problème des IST demeurerait. C'est pourquoi les règles du safer sex doivent continuer de s'appliquer.

- Même si toutes les personnes contaminées par le VIH dans les pays en développement savaient qu'elles étaient infectées, il n'y aurait pas assez de médicaments (abordables) pour toutes celles qui en ont besoin.

\section{La déclaration de la CFPS est valable et se base sur des données scientifiques}

Dans sa déclaration [2] du 30 janvier 2008, la Commission fédérale pour les problèmes liés au sida met en exergue que les individus chez qui un TAR [3] a réussi ne transmettent pas le virus lors de rapports sexuels. Elle indique que le risque de transmission du VIH lors de rapports sexuels respectant les règles précitées est bien inférieur à 1:100000. Une étude randomisée contrôlée a, depuis, confirmé cette déclara- 


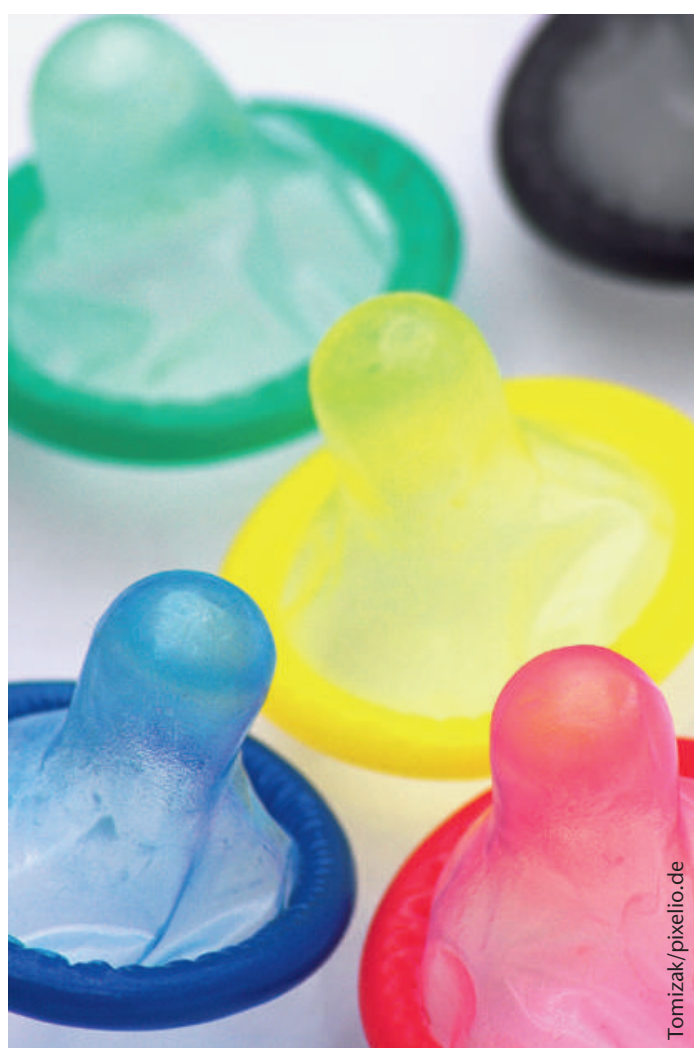

La Suisse a décidé de s'engager de manière délibérée dans la stratégie d'apprentissage. Chaque individu est responsable de se protéger du VIH.

tion [4]. Un risque résiduel n'est scientifiquement jamais exclu mais, jusqu'à aujourd'hui, aucune transmission du VIH n'a pu être prouvée dans les conditions mentionnées par la CFPS.

\section{Les HSH sont particulièrement touchés par le VIH}

Les derniers chiffres le montrent: il y a eu en 2011 presque autant de diagnostics du VIH parmi les hommes ayant des relations sexuelles avec d'autres hommes (HSH) que chez les hétérosexuels. Cependant, les homosexuels sont moins nombreux. On estime les HSH à environ 100000 personnes. Ce groupe est ainsi beaucoup plus touché que celui des hétérosexuels. La stratégie de l'OFSP visant à accroître les efforts de prévention sur les groupes touchés par VIH est judicieuse et, comme le montrent les chiffres, efficace.

\section{Conclusion}

La CFSS et la plupart des experts européens rejettent la démarche venue des Etats-Unis consistant à vou- loir tester tous les individus. Plutôt que de se soumettre à un test annuel, il est beaucoup plus important pour le patient de passer un test s'il présente les symptômes d'une primo-infection. L'infectiosité est alors extrêmement élevée et il est crucial d'obtenir rapidement un diagnostic. Malheureusement, celuici est encore bien souvent omis. La CFSS préfèrerait donc que le test du VIH soit effectué de manière ciblée, plutôt que voir critiquée la stratégie d'apprentissage, qui a fait ses preuves en Suisse. Fondé, le programme national VIH/IST a été élaboré par de nombreux experts renommés issus de divers domaines. Il propose une stratégie de prévention remarquable. Les tentatives consistant à remplacer cette stratégie, largement acceptée, par des idées de prévention individuelles et guidées par des objectifs flous sont douteuses. En tant que président de la CFSS, je vous invite à lire l'article sur le test du VIH dans le Bulletin 2011 de l'OFSP [5], et notamment la partie concernant le Provider-Initiated Counselling and Testing (PICT).

\section{Références}

1 Recommandations de la CCT sur le début d'une thérapie: www.bag.admin.ch/dokumentation/ publikationen/01435/11505/index.html?lang=fr\&dow nload=NHzLpZig7t,lnp6I0NTU04212Z6ln1ae2IZn4Z2 qZpnO2Yuq2Z6gpJCKdIJ8fWym162dpYbUzd,Gpd6e mK2Oz9aGodetmqaN19XI2IdvoaCUZ,s-

2 Déclaration de la Commission fédérale pour les problèmes liés au sida, devenue aujourd'hui la Commission fédérale pour la santé sexuelle (CFSS).

Vernazza P, Hirschel B, Bernasconi E, Flepp M. Les personnes séropositives ne souffrant d'aucune autre MST et suivant un traitement antirétroviral efficace ne transmettent pas le VIH par voie sexuelle (30.1.2008) www.saez.ch/docs/saez/archiv/fr/2008/2008-05/2008 05-089.PDF et

Vernazza P. La prévention du sida devient plus simple, mais aussi plus complexe!

www.saez.ch/docs/saez/archiv/fr/2008/2008-05/ 2008-05-085.PDF

3 Pour réussir, un TAR doit respecter différents critères: le traitement doit être suivi scrupuleusement et contrôlé régulièrement par le médecin; la charge virale se situe en-dessous du seuil de détection depuis au moins six mois et la personne séropositive n'est atteinte d'aucune autre IST.

4 Cohen et al. Prevention of HIV-1 Infection with Early Antiretroviral Therapy. N Engl J Med 2011;365:493505

5 Informations sur les Provider-Initiated Counselling and Testing (PICT) et sur les symptômes pouvant révéler une infection au VIH: www.bag.admin.ch/ hiv_aids/12472/12476/12479/index.html?lang=fr 